Revista Brasileira de Meteorologia, v.27, n.3, 291 - 306, 2012

\title{
VERIFICACIÓN DE LOS PRONOSTICOS DEL MODELO BRAMS CENTRADO EN LA REGION SUBTROPICAL DE SUDAMERICA.
}

\author{
YANINA GARCÍA SKABAR ${ }^{1,2,3,4}$, PAOLA SALIO ${ }^{4,5,6}$, MATILDE NICOLINI $^{4,5,6}$ \\ ${ }^{1}$ Servicio Meteorológico Nacional, Argentina. \\ ${ }^{2}$ CONICET,Argentina \\ ${ }^{3}$ Universidad de Buenos Aires (UBA), Facultad de Agronomía, Buenos Aires, Argentina. \\ ${ }^{4}$ UMI-Instituto Franco Argentino sobre Estudios del Clima y sus Impactos, Buenos Aires, Argentina. \\ ${ }^{5}$ UBA, Facultad de Ciencias Exactas y Naturales, Departamento de Ciencias de la Atmósfera y los Océanos, \\ Argentina \\ ${ }^{6}$ Centro de Investigaciones del Mar y la Atmósfera, CONICET-UBA, Argentina. \\ yanina@smn.gov.ar,salio@cima.fcen.uba.ar,nicolini@cima.fcen.uba.ar
}

\section{RESUMEN}

El objetivo del presente trabajo es realizar una verificación de los pronósticos generados en el Departamento de Ciencias de la Atmósfera y los Océanos de la Universidad de Buenos Aires con el modelo Brazilian Regional Atmospheric Modeling System(BRAMS). Utilizando dicho modelo, desde 2006, se realizan dos pronósticos al día por un plazo de 72 horas en dos retículas anidadas con una resolución horizontal de 80 y $20 \mathrm{~km}$. La retícula de mayor resolución abarca Paraguay, Uruguay, Sur de Brasil y Bolivia y el centro y norte de Argentina y Chile.

En este trabajo se realiza la verificación sobre el dominio de mayor resolución para el período desde abril de 2006 a diciembre de 2008. El interés se centra en verificar los pronósticos de precipitación, y de los perfiles verticales de temperatura, humedad y viento en las localidades donde se dispone de datos de radiosondeos. La verificación de los pronósticos de precipitación se realiza mediante estadísticos calculados frente a las observaciones convencionales disponibles y frente a estimaciones de precipitación CMORPH. Los resultados son similares a los obtenidos en la región con modelos de aproximadamente la misma resolución. Además se identifican las deficiencias principales de los pronósticos lo que permitirá realizar ajustes en la configuración del mismo para mejorar su desempeño. Palabras clave: pronóstico del tiempo, modelado numérico, verificación, BRAMS

\section{ABSTRACT: BRAMS FORECAST VERIFICATION OVERA REGION OF SUBTROPICAL SOUTHAMERICA}

The aim of this work is to verify the forecasts generated in the Department of Atmospheric Sciences and Ocean, University of Buenos Aires with the Brazilian model Regional Atmospheric Modeling System (BRAMS). Since year 2006, every day two different forecasts that extend for 72 hours have been performed using two nested grids with an horizontal resolution of 80 and $20 \mathrm{~km}$. The higher resolution domain covers Paraguay, Uruguay, southern Brazil and Bolivia and the central and northern part of Argentina and Chile.

This paper presents the results of the verification of the model in the higher resolution domain for the period April 2006 to December 2008. The focus is in the precipitation and vertical profiles of temperature and wind forecasts in the locations where soundings are available. Verification of precipitation forecast is based on statistics computed against observations and precipitation estimates from CMORPH. The results are close to those obtained in the region with models of similar resolution. It is also possible to identify the main forecast deficiencies, contributing to future adjustments in its configuration to improve the model performance.

Keywords: weather forecast; numerical modeling, verification, BRAMS. 


\section{INTRODUCCIÓN}

En la actualidad es indiscutible la utilidad de los pronósticos numéricos como herramienta a la hora de realizar un pronóstico. A la vez resulta indispensable realizar una verificación de los pronósticos numéricos con el fin de brindar a los usuarios una medida de su exactitud y detectar las deficiencias principales de los mismos para así poder realizar las modificaciones que sean necesarias para mejorar la calidad del pronóstico.

En las últimas décadas, debido al avance en los medios computacionales, modelos de mesoescala tales como el Weather Research and Forecasting (WRF) del National Centers for Environmental Prediction/ National Centers for Environmental Prediction (NCEP/NCAR), el ETA del NCEP y el Brazilian Regional Atmospheric Modeling System (BRAMS) se utilizan con el fin de generar pronósticos regionales en tiempo real para la región sur de Sudamérica. Diferentes versiones del modelo ETA se han implementado en forma operativa, con una resolución aproximada de $20 \mathrm{~km}$ en el Centro de Previsao de Tempo e Estudos Climaticos, Brasil (CPTEC) y en el Servicio Meteorológico Nacional, Argentina (SMN). El modelo WRF, en su versión ARW 2.0 (http://wrf.cima.fcen.uba.ar/), y el modelo BRAMS, en su versión BRAMS-3.2 (http://www.bramsuba. com.ar/), se utilizan rutinariamente con fines de pronóstico experimentales a corto plazo en el Centro de Investigaciones del Mar y la Atmósfera (CIMA) y en el Departamento de Ciencias de la Atmósfera y los Océanos (DCAO) de la Universidad de Buenos Aires (UBA), respectivamente. También se utiliza el modelo BRAMS en forma rutinaria en la Universidad de San Pablo, Brasil (USP). El uso de este tipo de modelos más sofisticados en la región ha representado un avance en la precisión de los pronósticos. Se puede ver una comparación entre los diferentes modelos en http://www.master.iag.usp.br.

En particular, el presente trabajo se centra en la evaluación de los pronósticos generados con el modelo BRAMS, que es un modelo numérico multipropósito que fue diseñado para simular circulaciones atmosféricas que van desde escalas hemisféricas a torbellinos de la capa límite planetaria. Se ha aplicado en mayor medida para simular fenómenos de meso y microescala. El modelo BRAMS, en su versión 3.2 desarrollado en conjunto por ATMET (Atmospheric, Meteorological and Environmental Technologies), IME-IAG/USP (Instituto de matemática y estadística -Instituto de Astronomía, Geofísica y Ciencias Atmosféricas/Universidad de San Pablo), y CPTEC es una adaptación de la versión 5.04 de RAMS (Regional Atmospheric Modeling System) desarrollado inicialmente en la Universidad Estatal de Colorado. Las características principales del modelo se describen en Freitas et al. (2009) y en la página web del modelo (www.brams.cptec.inpe.br).
Hasta el momento se han realizado evaluaciones del desempeño del modelo en casos de estudio en Argentina para diferentes situaciones sinópticas utilizando distintas parametrizaciones, resoluciones y condiciones iniciales y de contorno (Saulo et al., 2007; Nicolini et al., 2002; Salio et al., 2006; Ruiz et al., 2006; Nicolini et al., 2005a; Nicolini et al., 2005b; Salio et al., 2009). En dichos trabajos se ha encontrado un desempeño satisfactorio del mismo para simular y pronosticar casos con precipitación intensa, un caso de granizo, un caso de nevada en el sudeste bonaerense, un caso de convección organizada, un caso de un tornado en Resistencia y la inundación del 2007 en el sur de Santa Fe y Entre Ríos, Argentina. Asimismo, se generó para la temporada cálida 20022003, un conjunto de análisis enriquecidos asimilando todos los datos obtenidos durante el South American Low-Level Jet Experiment (SALLJEX) (García Skabar y Nicolini, 2009) y se estudió el impacto de la asimilación de datos en el pronóstico para el mismo período (García Skabar, 2008).

Si bien se han realizado evaluaciones del desempeño del modelo en casos de estudio o en períodos cortos en la región de interés, el objetivo del presente trabajo es realizar una verificación sobre la región subtropical de Sudamérica en un período más extenso, de aproximadamente 3 años de los pronósticos generados con el modelo BRAMS. Resulta de interés, especialmente para fines operativos, poder contar con una evaluación del desempeño de los pronósticos del modelo sobre la región. La verificación se realiza en todo el período sin estudiar en detalle los mecanismos que actúan en los diferentes casos particulares. Se centra principalmente en una evaluación general de los pronósticos de precipitación, y además se estudia el desempeño del pronóstico de los perfiles verticales de temperatura, humedad y viento en las localidades de la región donde se dispone de datos de radiosondeos.

Este trabajo se organiza del siguiente modo, en la sección 2 se presenta una descripción de las características principales de la configuración del modelo, los datos y las metodologías de verificación utilizadas. En la sección 3 se muestran y discuten los resultados obtenidos y finalmente en la sección 4 se exponen las conclusiones.

\section{DATOS Y METODOLOGÍA}

\subsection{Descripción del modelo BRAMS}

La configuración del modelo se ha seleccionado en base a diferentes experimentos numéricos donde se fueron variando las opciones físicas para casos específicos y se eligió la que más favorecía al pronóstico en la región de estudio. Una configuración similar ha sido utilizada en trabajos anteriores (García Skabar y Nicolini, 2009, Borque et al., 2010, Nicolini y 
García Skabar, 2011). Se configuró el modelo con dos retículas anidadas de 80 y $20 \mathrm{~km}$ de resolución, utilizando un anidado interactivo. Los dominios que abarcan las retículas se muestran en la Figura 1. El modelo es no hidrostático y utiliza una proyección estereográfica polar. Se utilizan 9 niveles de suelo y 29 niveles verticales en la atmósfera, donde dentro de los primeros $12 \mathrm{~km}$ se cuenta con 18 niveles y el tope del modelo se ubica aproximadamente en $23 \mathrm{~km}$. Se utiliza la coordenada vertical sigma Z (Tripoli y Cotton, 1982). En ambas resoluciones se activan las parametrizaciones de la convección tanto poco profunda (Souza y Silva, 2002) como profunda. En el caso de la convección profunda se utiliza la parametrización de Grell con el cierre de Grell (Grell, 1993; Grell y Devenyi, 2002). Para la representación de la microfísica se emplea el esquema "bulk water" de un momento que considera una subdivisión del agua en ocho especies diferentes (Walko et al., 1995). Se trabaja con la parametrización de la radiación de Chen y Cotton (1983). La turbulencia subreticular se simula con los esquemas de Mellor y Yamada (1982) en la vertical y de Smagorinsky (1963) en la horizontal.

El modelo incorpora datos de topografía con resolución de aproximadamente $1 \mathrm{~km}$ provenientes del United States Geological Survey (USGS), de uso de suelo o cobertura vegetal con resolución de $1 \mathrm{~km}$ del International Geosphere - Biosphere Programme (http://edcdaac.usgs.gov/glccc) y de tipos de suelo con resolución de $5 \mathrm{~km}$ de FAO/UNESCO (http://daac. gsfs.nasa.gov). Se incorporan también campos semanales de temperaturas de la superficie del mar provenientes de National Centers for Environmental Prediction (NCEP), en su versión OI.v2 con resolución de $100 \mathrm{~km}$ que se describen en Reynolds et al., 2002. Por otro lado, se incorporan campos de humedad de suelo diarios los cuáles pueden ser incorporados al modelo como condición inicial. Dichos campos de humedad del suelo se generan con datos provenientes de un modelo que la evalúa en base a la precipitación observada por sensores remotos (Gevaerd y Freitas, 2006).

Se realizan dos pronósticos al día inicializados a las 00 y 12 UTC por un plazo de 72 horas y con una resolución temporal de 1 hora. Se utilizan como condición inicial y de borde los análisis y pronósticos operativos Global Forecast System (GFS) provenientes de National Oceanic and Atmospheric Administration (NOAA)/NCEP con una resolución horizontal de $1^{\circ}$, vertical de 26 niveles y temporal de 6 horas. Tanto las condiciones iniciales como las de borde son incluidas solamente en la retícula de menor resolución horizontal.

Dado que el modelo no se utiliza con fines operativos, existen días en los que el pronóstico no se pudo realizar por motivos relacionados con fallas técnicas (el porcentaje de prónosticos faltantes es menor al 5\%) y por otro lado durante el receso estival del mes de enero el pronóstico del modelo no está disponible. No se cuenta con la infraestructura suficiente como para realizar una recuperación de los pronósticos faltantes,

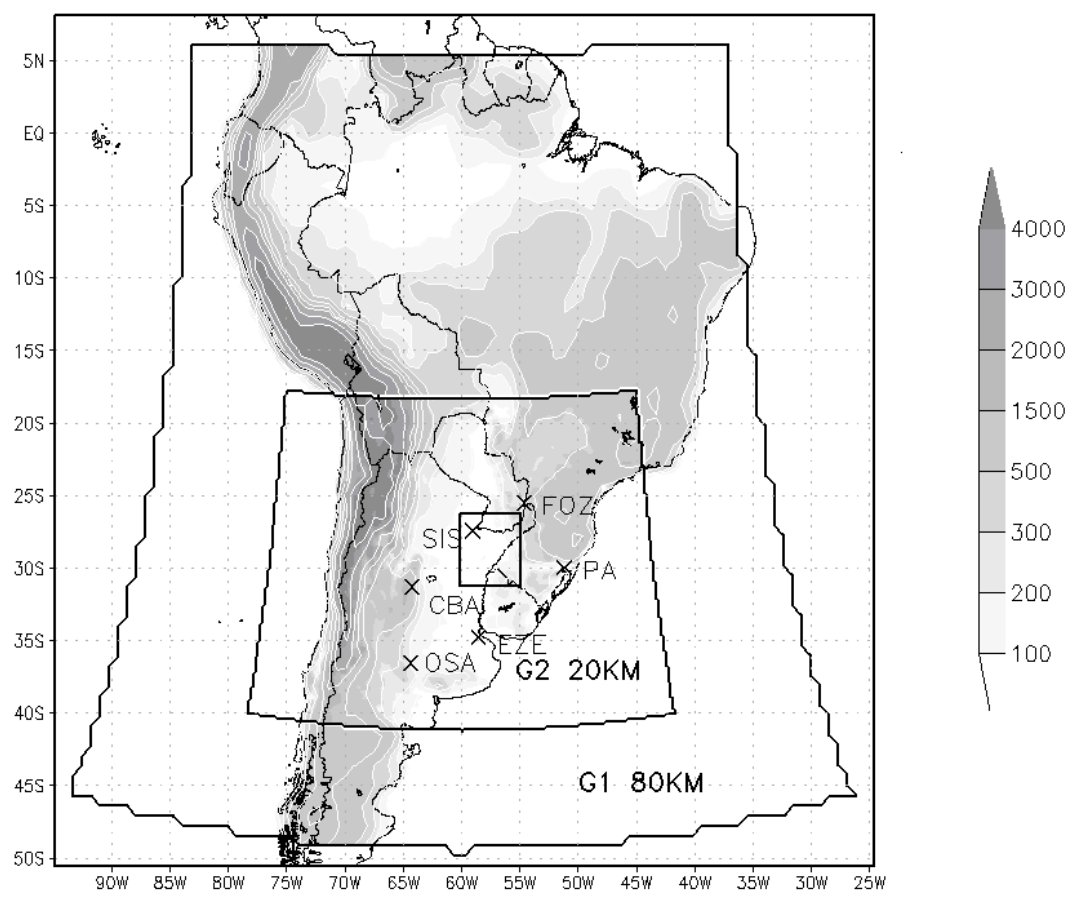

Figura 1 - Dominios utilizados, topografía sombreado. Se muestran con cruces la ubicación de las estaciones de radiosondeos utilizadas en la verificación de los perfiles verticales. Abreviaturas: EZE:Ezeiza, OSA:Santa Rosa, DOZ: Mendoza, SIS: Resistencia, CBA: Córdoba, FOZ:Foz de Iguazú, PA:Porto Allegre. El rectángulo representa el área donde se promedia la precipitación. 
además de la dificultad adicional que representa recuperar los campos para inicializar el modelo.

En el presente trabajo se realiza una verificación del desempeño del pronóstico del modelo en el dominio de mayor resolución en el periodo de abril de 2006 a diciembre de 2008 inclusive. Se consideran tanto los pronósticos inicializados a las 00 como a las 12 UTC.

\subsection{Datos utilizados y metodología de verificación}

\subsubsection{Perfiles verticales}

Se utilizan para la verificación las observaciones de radiosondeos disponibles en la región. Se considera en el periodo de estudio las localidades de Ezeiza, Santa Rosa, Resistencia y Córdoba de Argentina y Puerto Alegre y Foz de Iguazú de Brasil, cuya ubicación se muestra en la Figura 1. En la Tabla 1 se muestra la cantidad de radiosondeos disponibles en cada localidad todos ellos en el horario de 1200 UTC, lo que permite verificar los pronósticos a 24, 48 y 72 horas inicializados a las 1200 UTC y los pronósticos a 12, 36 y 60 horas inicializados a las 0000 UTC. El número de datos se limita a los días en que el modelo estuvo disponible.

La verificación frente a observaciones de radiosondeos se realiza mediante el cálculo de la raíz cuadrada del error cuadrático medio (RMSE) y los desvíos (BIAS) para la temperatura y las componentes del viento en 11 niveles de presión $(1000,925,850,700,500,400,300,250,200,150$ y 100 $\mathrm{hPa}$ ) limitando el cálculo a los niveles de presión superiores o iguales a 300hPa para la relación de mezcla. Se define el RMSE $\mathrm{y}$ el BIAS para una variable $\mathrm{X}$ como:

$$
\begin{aligned}
R M S E & =\left[\frac{1}{N} \sum_{i=1}^{N}\left(X p^{i}-X o^{i}\right)^{2}\right]^{1 / 2} \\
B I A S & =\frac{1}{N} \sum_{i=1}^{N}\left(X p^{i}-X o^{i}\right)
\end{aligned}
$$

donde $\mathrm{N}$ es el número total de observaciones, el subíndice $\boldsymbol{p}$ significa pronosticado y $\boldsymbol{o}$ observado. El sondeo pronosticado se estima como el promedio de 9 puntos alrededor del punto de retícula del modelo más cercano a la localidad de observación.

\subsubsection{Precipitación}

En el caso de la precipitación se utiliza la información convencional de precipitación acumulada de 24 horas a las 12UTC proveniente de numerosas instituciones de diferentes países: Servicio Meteorológico Nacional, Administración Provincial del Agua (Chaco), Secretaría de Recursos Hídricos
Tabla 1 - Número de radiosondeos utilizados en la verificación en cada localidad.

\begin{tabular}{|l|c|}
\hline Estación & $\mathrm{N}^{\circ}$ de datos \\
\hline Ezeiza & 718 \\
\hline Resistencia & 506 \\
\hline Santa Rosa & 517 \\
\hline Córdoba & 452 \\
\hline Foz de Iguazú & 570 \\
\hline Porto Allegre & 638 \\
\hline
\end{tabular}

de la Nación, Universidad de La Punta (San Luis), Autoridad Interjurisdicional del Aguas (Neuquén), Comisión Técnica Mixta Salto Grande, Instituto Nacional de Tecnología Agropecuaria y Bolsa de Cereales de Argentina; Dirección Nacional de Meteorología de Uruguay y de Paraguay, Centro de Previsão de Tempo e Estudos Climáticos de Brasil; y Climate Diagnostics Center (CDC)-NOAA de E.E.U.U.. Los datos disponibles se promedian espacialmente en cajas centradas en los puntos de retícula de mayor resolución del modelo. Existen cajas que para algunas fechas en particular no tienen observaciones, las mismas no se utilizan en la verificación. Se considera como observación siempre que el dato este disponible aunque la precipitación observada sea cero. Por lo tanto, el número de días con observaciones en cada caja es diferente. En la Figura 2 se muestra para cada caja, el número de días en que el modelo estuvo disponible y a la vez existía alguna observación. Sobre esa cantidad de datos se realiza la verificación. Esta metodología difiere de las técnicas de interpolación ampliamente utilizadas para representar campos observados de precipitación tales como el kriging (Davies, 1973), donde por un lado la precipitación es suavizada y por otro se generan valores de precipitación en regiones donde no hay observaciones. Esto hace que la comparación realizada sea más estricta en el sentido que en los puntos donde no hay datos no se realizan las verificaciones, y en las cajas con datos no se realizan interpolaciones sino simplemente promedios. Esta metodología fue utilizada por Liebmann y Allured (2005) para generar una base de datos histórica en puntos de retícula para Sudamérica con 1 grado de resolución.

Si bien la red observada en la Figura 2 muestra una densidad superior a las redes operativas por los Servicios Meteorológicos Nacionales, presenta aún una cobertura espacial deficiente. Por ello, es necesario recurrir a fuentes de información alternativas como las estimaciones de precipitación mediante el uso de satélites. En este caso se utilizaron las estimaciones de precipitación CMORPH (Climate Prediction Center Morphing Technique) descriptas en Joyce et al. (2004), que cuentan con una resolución espacial de $8 \mathrm{~km}$ y temporal horaria. Cabe señalar que el CMORPH muestra en sus dos resoluciones disponibles 


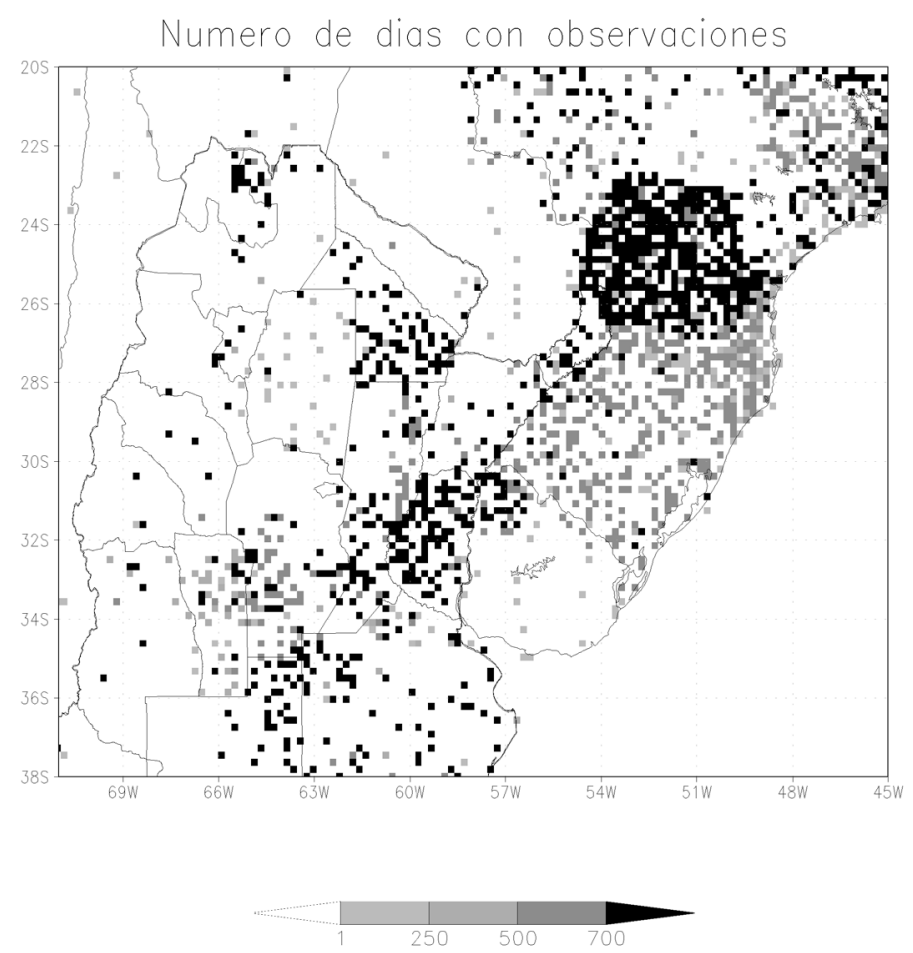

Figura 2 - Número de días con datos de precipitación en cada caja en el periodo disponible del modelo.

(8 y $25 \mathrm{~km}$ ) sobreestimaciones en los valores de precipitación asociados a eventos de convección profunda. Si bien las estimaciones de precipitación TRMM 3B42 version 6 (Huffman et al., 2007) presentan un mejor comportamiento en la región, especialmente en la detección y estimación de eventos asociados a convección profunda (Salio et al., 2010), se decidió utilizar las estimaciones CMORPH debido a su mayor resolución espacial. Se calculó, para las mismas cajas que se utilizaron en el caso de las observaciones convencionales, la precipitación acumulada de 24 horas a las 1200UTC, promediando todos los valores estimados dentro de cada caja.

La verificación de los pronósticos de precipitación se realizó por un lado con las observaciones convencionales y por otro con las estimaciones CMORPH, obteniendo diferentes estadísticos para cada caso.

La precipitación pronosticada se evaluó mediante los estadísticos Equitable Treath Score (ETS) y desvío (BIASPP) considerando todo el dominio de mayor resolución, siguiendo la definición de Mesinger (1996):

$$
\mathrm{ETS}=(\mathrm{H}-\mathrm{CH}) /(\mathrm{F}+\mathrm{O}-\mathrm{H}-\mathrm{CH}) \quad \text { con } \quad \mathrm{CH}=\mathrm{FxO} / \mathrm{N}
$$

\section{BIASPP $=\mathrm{F} / \mathrm{O}$}

donde: $\mathrm{F}$ es el número de puntos en los que se pronosticó precipitación, $\mathrm{O}$ es el número de puntos en los que se observó precipitación, H es el número de puntos en los que se pronosticó y se observó precipitación y $\mathrm{N}$ es el número de puntos con datos en todo el dominio de verificación. Estos mismos estadísticos fueron empleados para la verificación de pronósticos de precipitación sobre el sur de Sudamérica por otros autores (Saulo y Ferreira, 2003; Saulo et al., 2008, entre otros).

El ETS resulta proporcional a la cantidad de aciertos mientras que el BIASPP compara el tamaño de las áreas donde precipitó, sin importar la ubicación de la misma. Los valores de BIASPP mayores/menores a uno indican que el área donde se pronóstico precipitación es mayor/menor al área donde se observó. En un pronóstico perfecto el ETS y el BIASPP serían iguales a uno.

Tanto el ETS como el BIASPP se calcularon para ocho umbrales de precipitación $(0.25,2.50,6.30,12.7,19.0,25.4$, 38.1 y $50.8 \mathrm{~mm}$ ) que son los más utilizados en la literatura (Ebert et al.,2007 y referencias allí citadas). Se considera la precipitación acumulada en el período de 24 horas acumulando a las 1200 UTC debido a que es el intervalo de tiempo en el que la mayor parte de la información está disponible. Ambos estadísticos se calcularon por separado para los dos conjuntos de datos: observados y estimaciones de precipitación CMORPH.

Para realizar una verificación espacial del pronóstico de precipitación se calcularon campos de RMSE y BIAS respecto a los campos de precipitación estimada CMORPH y 
a las observaciones convencionales para el período completo. Asimismo se analizaron los campos de RMSE y BIAS por separado para el semestre frío y cálido, definidos como los meses de abril a septiembre y octubre a marzo respectivamente, con el fin de analizar si existe alguna estacionalidad en la distribución de los errores.

Por otro lado se calculó la frecuencia porcentual de días en los que la precipitación, según los pronósticos, las observaciones convencionales y las estimaciones CMORPH, superó un umbral de $1 \mathrm{~mm}$ y $50 \mathrm{~mm}$. El primer umbral señala los aciertos en el pronóstico de lluvia o no lluvia, mientras que el umbral de 50 $\mathrm{mm}$ permite evaluar el pronóstico de eventos más extremos.

\section{RESULTADOS}

\subsection{Perfiles termodinámicos y de viento}

En la Figura 3 se muestran los perfiles de RMSE para las diferentes variables en Ezeiza. En dicha figura se comparan los errores en los diferentes tiempos de pronóstico. Se presentan los valores para los pronósticos a 12 y $60 \mathrm{hs}$ de la corrida inicializada a las 00UTC y los correspondientes a 24 y $72 \mathrm{hs}$ de la corrida inicializada a las 12 UTC. Se puede observar, como es de esperar, que a medida que aumenta el tiempo de pronóstico se produce un aumento en los valores de RMSE,
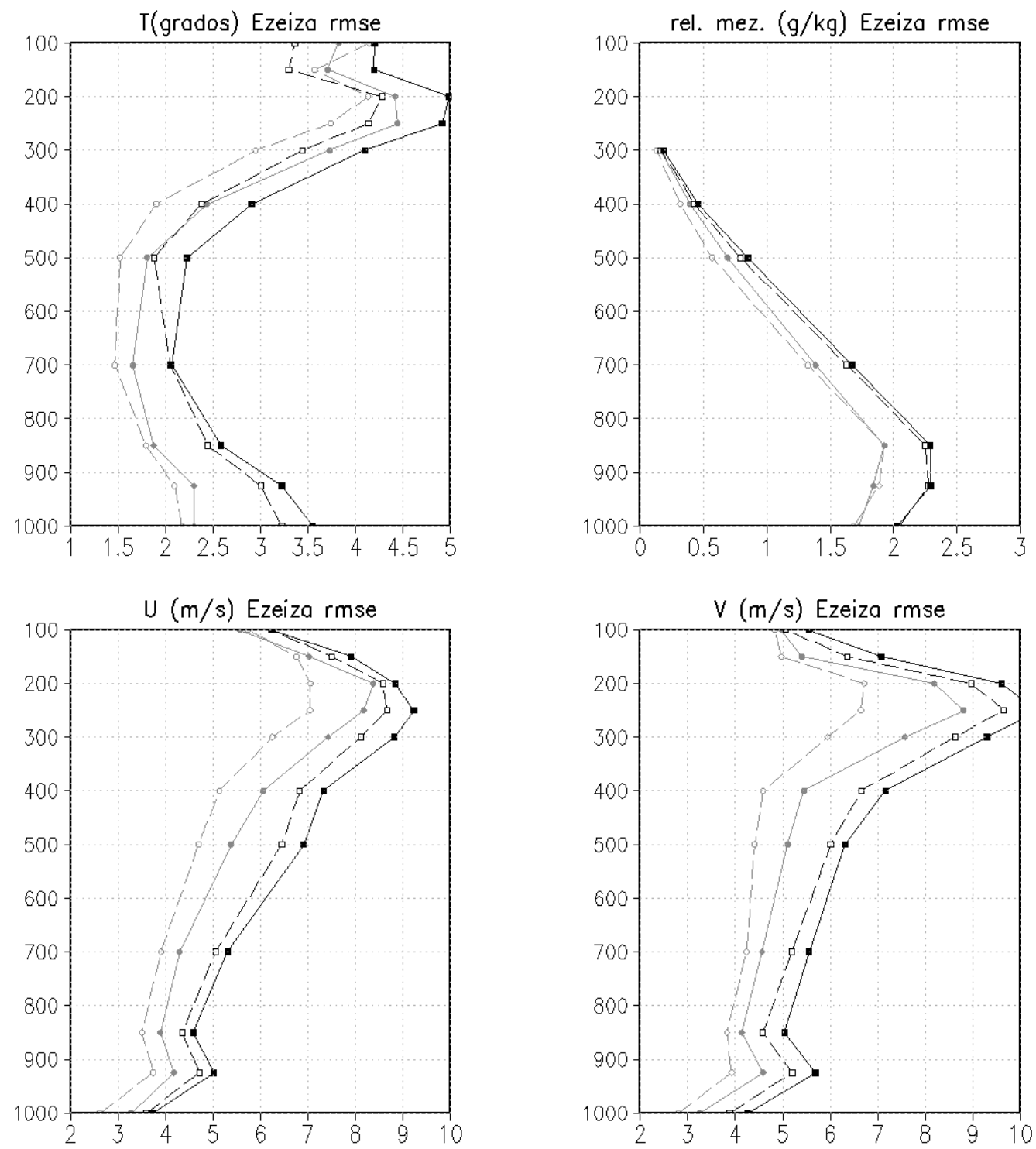

Figura 3 - Perfiles verticales de RMSE para Ezeiza, Argentina. De izquierda a derecha: paneles superiores Temperatura $\left({ }^{\circ} \mathrm{C}\right)$ y relación de mezcla $(\mathrm{g} /$ $\mathrm{kg}$ ); paneles inferiores: componente zonal y meridional del viento $(\mathrm{m} / \mathrm{s})$. Las líneas cortadas y llenas corresponden a las corridas inicializadas a las 00 UTC y a las 12 UTC respectivamente. Las líneas grises corresponden a los pronósticos a 12 y 24 hs y las líneas negras a los pronósticos a 60 y 72 hs. 
indicando una disminución en la precisión del pronóstico. Se muestran solamente el mejor (12 y $24 \mathrm{hs}$ de pronóstico) y el peor (60 y $72 \mathrm{hs}$ de pronóstico) valor obtenido para cada corrida, obteniéndose valores intermedios para los pronósticos a 30 y 48 hs de los pronósticos inicializados a las 0000 y 1200 UTC respectivamente (no se muestra). Un perfil similar en el error se repite en todos los tiempos de pronóstico. Se observa que tanto para la temperatura como para las componentes del viento el error aumenta con la altura, en cambio para la humedad el mayor error se produce en niveles bajos. Estas variaciones verticales del RMSE para las diferentes variables están asociadas en parte a la variación en la magnitud de la variable. Si bien no se muestran los errores al mismo tiempo de pronóstico debido a que solamente se dispone de radiosondeos a las 1200 UTC, ya no se observa un mejor desempeño del modelo inicializado a las 1200 UTC respecto al inicializado a las 0000 UTC, como señalaban en Saulo et al. (2001). Dicha diferencia en el desempeño era justificada por la mayor cantidad de datos convencionales incorporados en el análisis de 12 UTC. La gran cantidad de datos satelitales asimilados en los análisis hacen que la calidad de los mismos sea similar a las 0000 y a las 1200 UTC. Se muestra la figura para la localidad de Ezeiza a modo de ejemplo, pero el mismo comportamiento respecto a los tiempos de pronóstico se obtiene para las otras localidades analizadas (no se muestra).

En las Figuras 4 y 5 se presentan los perfiles de RMSE y BIAS, respectivamente, para las diferentes variables y localidades, solamente para el pronóstico a 24 horas para el período completo, dado que la variación de los errores para los diferentes tiempos de pronóstico es similar a la que se mostró para la localidad de Ezeiza. No se presentan los perfiles para la localidad de Santa Rosa debido a que son similares
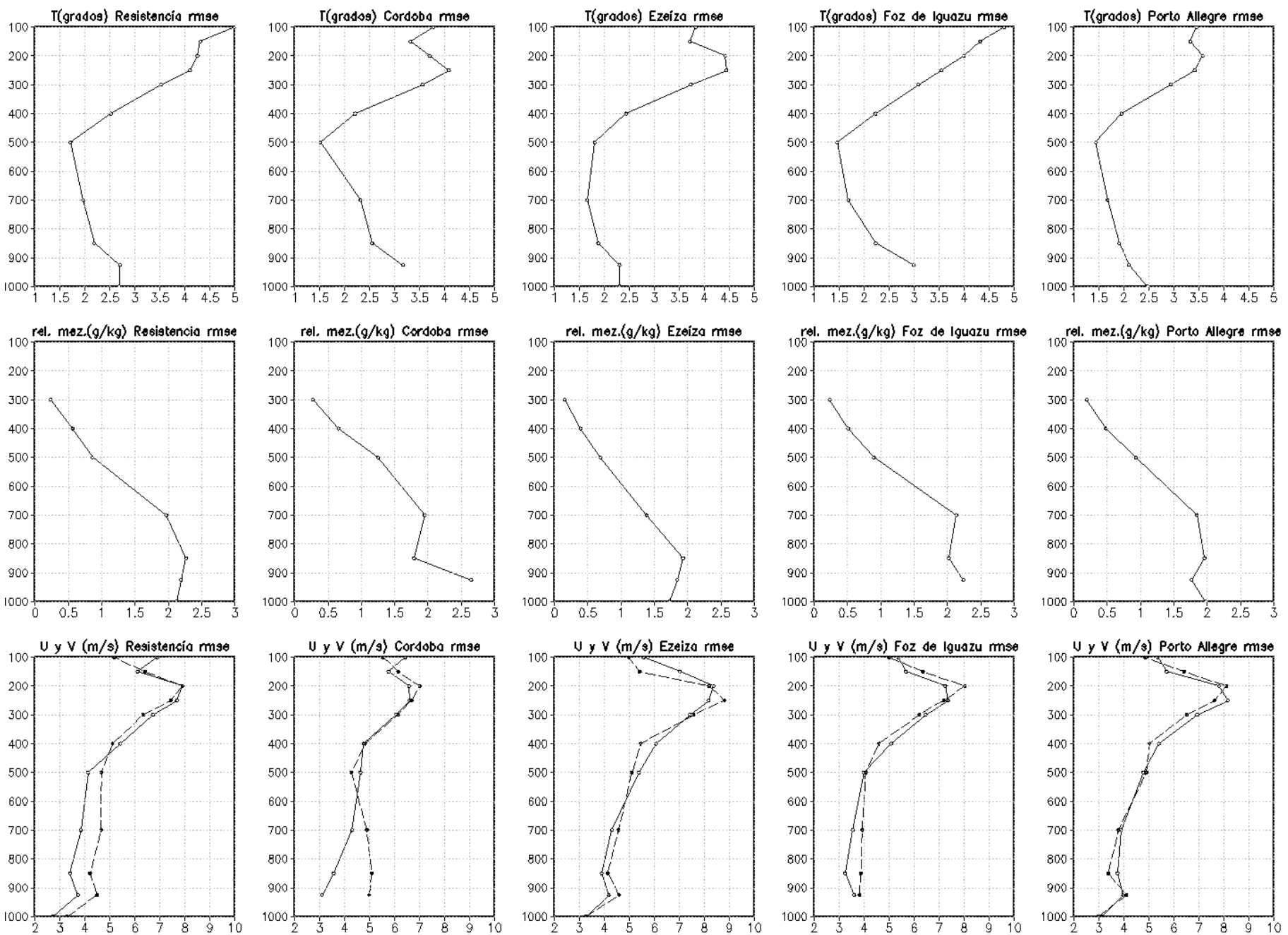

Figura 4 - Perfiles verticales de RMSE correspondientes al pronóstico a 24hs inicializado a las 12 UTC. De izquierda a derecha para Resistencia, Córdoba y Ezeiza, Argentina y Foz de Iguazú y Porto Allegre, Brasil. De arriba abajo para Temperatura $\left({ }^{\circ} \mathrm{C}\right)$, relación de mezcla $(\mathrm{g} / \mathrm{kg})$ y componente zonal y meridional del viento $(\mathrm{m} / \mathrm{s})$ (líneas llenas componente zonal y líneas cortadas componente meridional) 

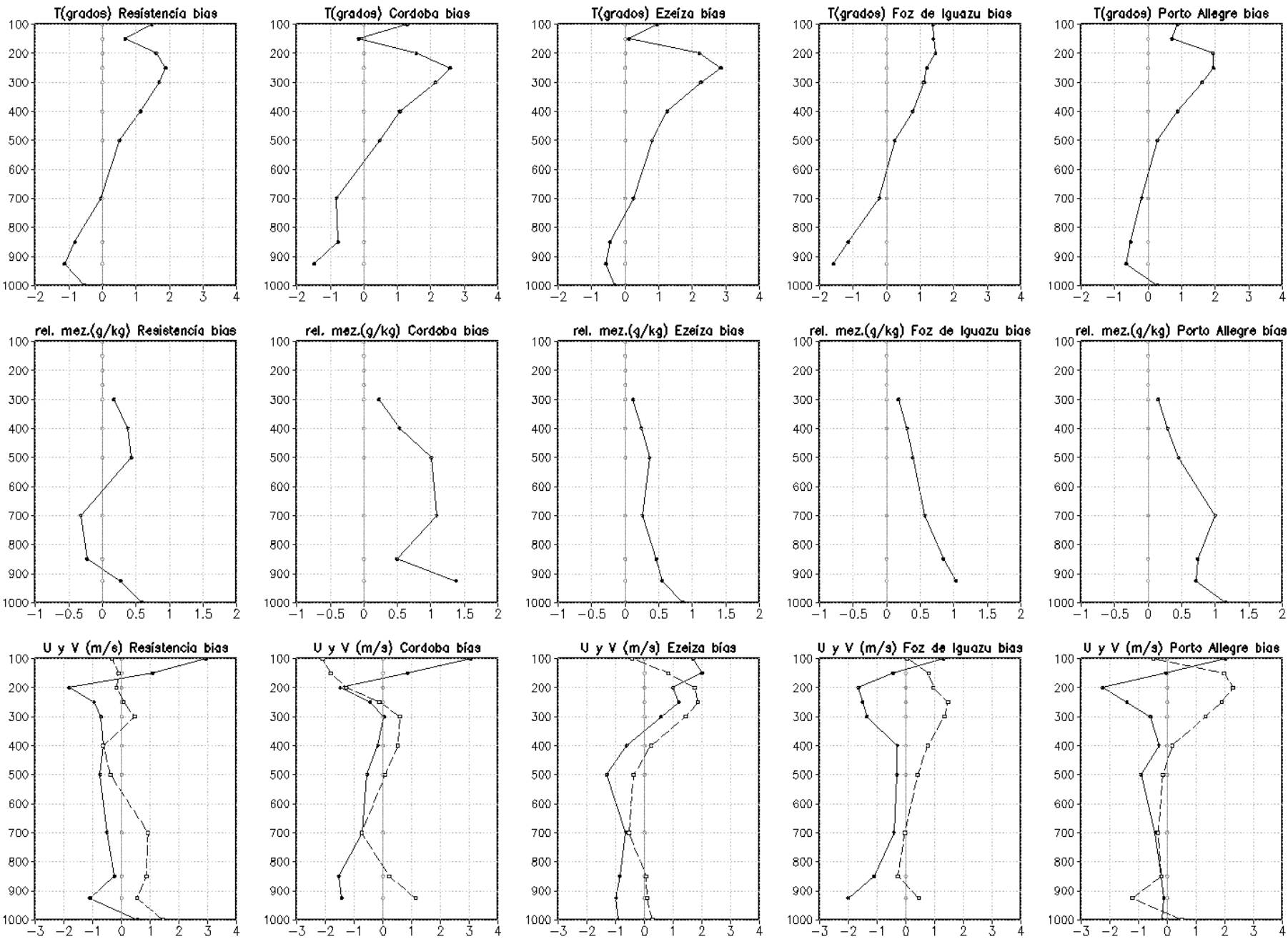

Figura 5 - Idem figura 4 para el BIAS.

a los resultados obtenidos para Ezeiza. Cabe señalar que la verificación se realiza frente a observaciones y no frente a análisis producidos por otros modelos, lo cuál representa una mayor exigencia y a la vez resulta más difícil realizar comparaciones con otros trabajos en la región ya que en la mayor parte de estos casos se calculan los estadísticos frente a análisis producidos por modelos. Para todas las localidades se puede ver un aumento del error en ambas componentes del viento y la temperatura en los niveles altos, lo que puede estar justificado en parte debido a la ubicación de la observación. Los sondeos estimados mediante el modelo representan el punto de retícula más cercano a la localidad de observación para todos los niveles, en cambio el globo del radiosonda, si bien es lanzado en un punto a medida que asciende se va desplazando del lugar y esto sucede en mayor medida en los niveles elevados donde eventualmente se encuentra la corriente en chorro. Por otro lado, es importante considerar la variación que existe con la altura en la magnitud de la variable. Por ejemplo en el caso de la componente zonal del viento su valor medio varía entre $0 \mathrm{~m} / \mathrm{s}$ en $1000 \mathrm{hPa}$ hasta $35 \mathrm{~m} / \mathrm{s}$ en los niveles donde el error es máximo (200 hPa). Es decir que el error si bien es mayor en niveles altos no representa más del 25\% del valor de la variable.

Las curvas de RMSE para la temperatura muestran un mayor error en niveles bajos asociado a la dificultad que presentan los modelos en general para representar la amplitud térmica diaria. Esto se puede ver en la comparación de diferentes modelos en la región con observaciones de temperatura de superficie (disponible la pagina web de Meteorología aplicada a sistemas de tiempo regional, www.master.iag.usp.br). Asimismo, a partir de los perfiles de BIAS (Figura 5) se puede ver que la temperatura es subestimada en niveles bajos y sobrestimada en niveles medios y altos, produciendo una estabilización del gradiente de temperatura vertical, lo que podría conducir a una disminución en la precipitación pronosticada.

Los perfiles de BIAS de la componente zonal del viento muestran una sobreestimación de los vientos del sector este o 
bien una subestimación del viento del sector oeste hasta el nivel de $200 \mathrm{hPa}$, que se repite en todas las localidades. En cambio la componente meridional del viento presenta comportamientos distintos en cada localidad. En particular en Resistencia se puede ver una subestimación del viento del sector norte en capas bajas hecho que ya fue constatado en trabajos previos (García Skabar, 2008; García Skabar y Nicolini, 2009).

Los perfiles de BIAS de relación de mezcla muestran una sobreestimación del contenido de vapor de agua en todos los niveles y para todas las localidades excepto para Resistencia donde en niveles bajos entre 900 y $700 \mathrm{hPa}$ se observa una subestimación de la misma. El perfil vertical observado medio en Resistencia (no se muestra) pone en evidencia la presencia de la corriente en chorro en capas bajas al este de los Andes (SALLJ). El SALLJ representa un efectivo mecanismo para el transporte de humedad de latitudes tropicales a subtropicales y tiene un rol fundamental en el ciclo hidrológico en el Sudeste de Sudamérica (Salio et al., 2002; Marengo et al., 2004; Berbery y Barros, 2002). Los perfiles de BIAS de la relación de mezcla y de la componente meridional del viento en Resistencia señalan cierta dificultad del modelo en representar el SALLJ, coherente con una subestimación en capas bajas tanto de la humedad como de la intensidad del flujo del sector norte. Este comportamiento podría conducir a una subestimación de la precipitación.
Es importante destacar que la magnitud de los RMSE y BIAS es similar en todas las localidades, mostrando que el modelo logra representar los perfiles de temperatura, humedad $\mathrm{y}$ viento con la misma calidad en localidades que se encuentran en el llano como en localidades afectadas por topografías más complejas como Córdoba que se encuentra en una zona serrana.

\subsection{Precipitación}

En la Figura 6 se presentan los valores de ETS y BIASPP obtenidos para la precipitación acumulada de 24 horas, para diferentes tiempos de pronóstico. Los valores obtenidos de ETS presentan un valor máximo de 0.4 para umbrales bajos de precipitación, lo que se encuentra en el rango de valores obtenidos en estudios previos en la región (Saulo et al., 2008). Al igual que en los perfiles verticales de temperatura y viento la calidad del pronóstico disminuye a medida que aumenta el tiempo de pronóstico. Asimismo los valores de ETS disminuyen a medida que aumenta el umbral de precipitación, señalando la dificultad para representar con exactitud la ocurrencia de casos de precipitación intensa. Se debe considerar que el número de casos en los que se registra precipitación intensa es menor que los casos en los que se observa precipitación débil. Es decir que la cantidad de datos que contiene la muestra sobre la que se esta
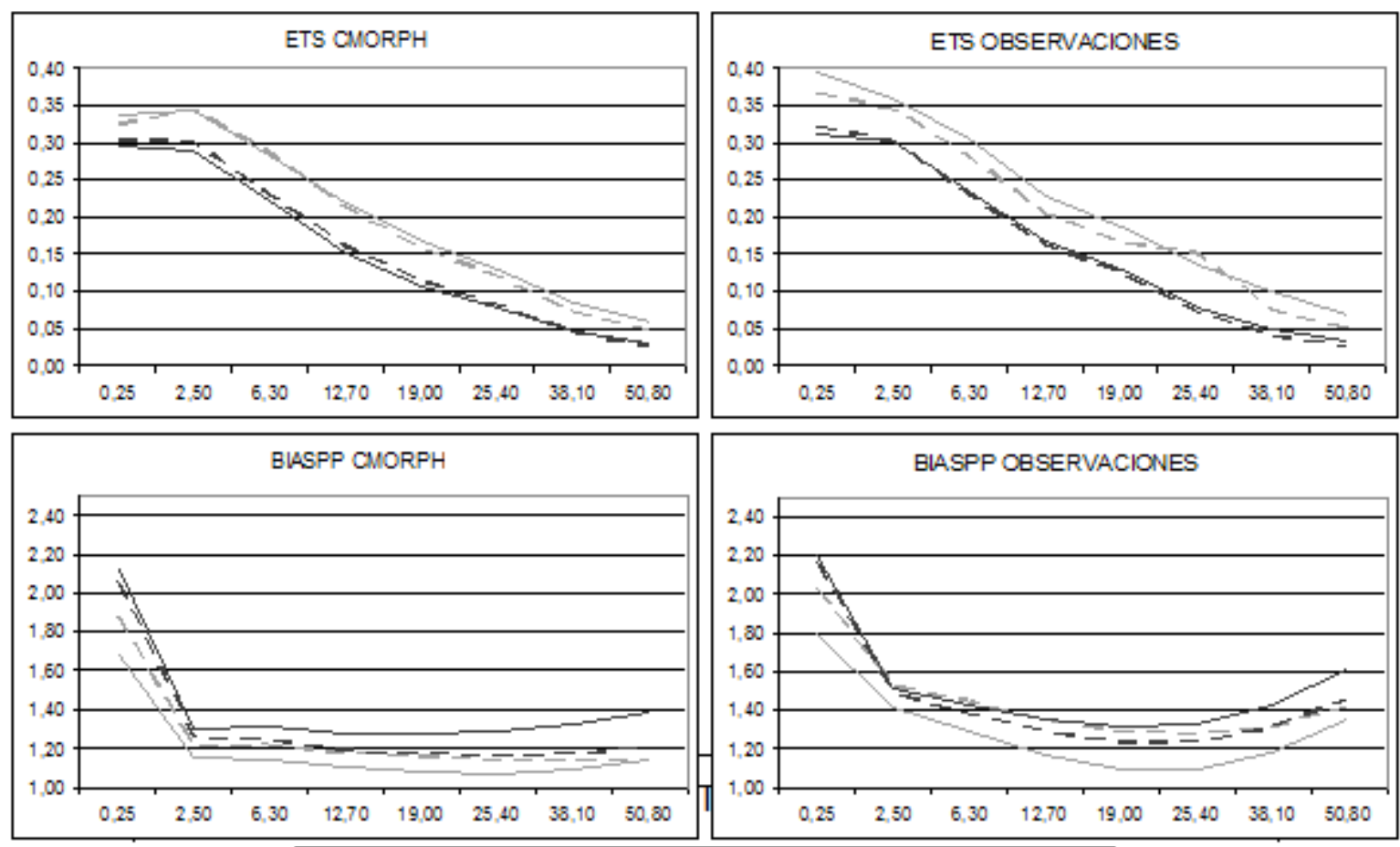

F24hs - F36hs -- F60hs

F72hs

Figura 6 - ETS y BIASPP de los pronósticos a 24, 36, 60 y 72 horas para los distintos umbrales de precipitación (mm). Paneles de la izquierda verificación con estimaciones CMORPH, paneles de la derecha con datos convencionales. 
verificando es menor cuanto mayor es el umbral de precipitación y por lo tanto disminuye la validez de los estadísticos. Por su parte, la frecuencia de tasas de precipitación más intensas también varía espacialmente sobre la región de estudio observándose tasas menos frecuentes hacia la Cordillera y hacia el sur del país donde el percentil del 95\% alcanza un acumulado de $20 \mathrm{~mm}$ en el centro del país (Robledo y Penalba, 2009).

Es importante destacar la dependencia de este tipo de estadísticos respecto de la información utilizada. Se observan diferencias en los resultados según el conjunto de datos utilizados especialmente en los valores obtenidos de BIASPP debido a la cobertura espacial de los mismos. Respecto a las observaciones convencionales, las curvas de BIASPP muestran que el pronóstico presenta una sobreestimación más importante del área con precipitación en umbrales bajos y en umbrales mayores a $25 \mathrm{~mm}$. Sin embargo al calcular el BIASPP respecto a la precipitación estimada de CMORPH la sobreestimación tan marcada para umbrales altos no se observa, lo que esta relacionado con la tendencia que presentan las estimaciones
CMORPH a sobrestimar la precipitación en la región (Ruiz, 2009; Salio et al., 2010).

Por otro lado, en la Figura 7 se muestran los campos de BIAS y RMSE de los pronósticos a 24 horas respecto a los datos CMORPH y respecto de las observaciones convencionales para el período total. En la figura se puede ver que el modelo subestima la precipitación en el Noreste de Argentina, y es en esta región donde los errores son más importantes, hecho que se relaciona con una subestimación del SALLJ (mencionada en la sección 3.1) y de la convergencia de humedad asociada al mismo. Ruiz (2009) muestra que las estimaciones CMORPH sobreestiman la precipitación en una temporada cálida especialmente en el Noreste de Argentina. Si bien las estimaciones presentan un error en la región se puede ver que la subestimación de la precipitación también se observa al comparar los pronósticos frente a las observaciones, aunque en la región donde el error es máximo existen muy pocos datos. También se observa que frente a las dos fuentes de observación el modelo sobrestima la precipitación en la región Sudeste del a) rmse total $24 \mathrm{hs}$ CMORPH

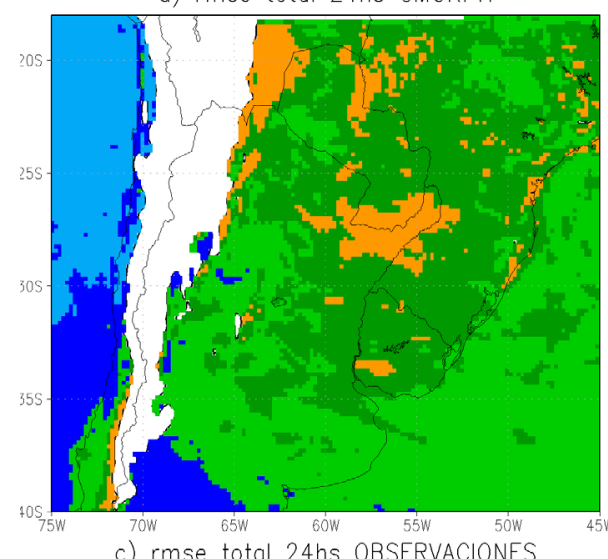
c) rmse total 24 hs OBSERVACIONES

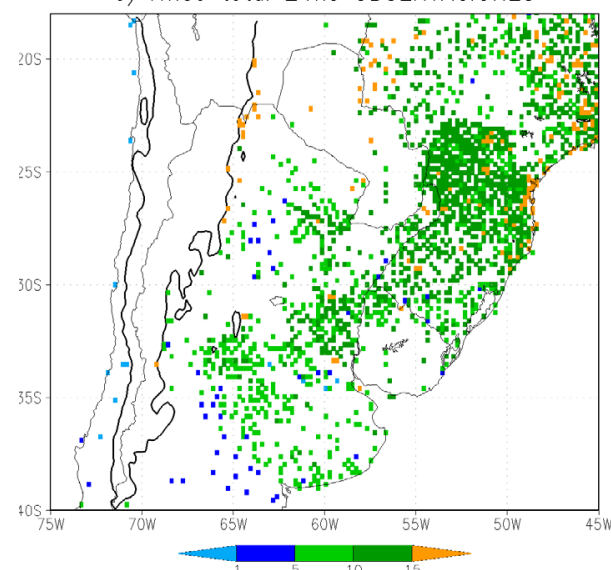

b) bios totol $24 \mathrm{hs}$ CMORPH

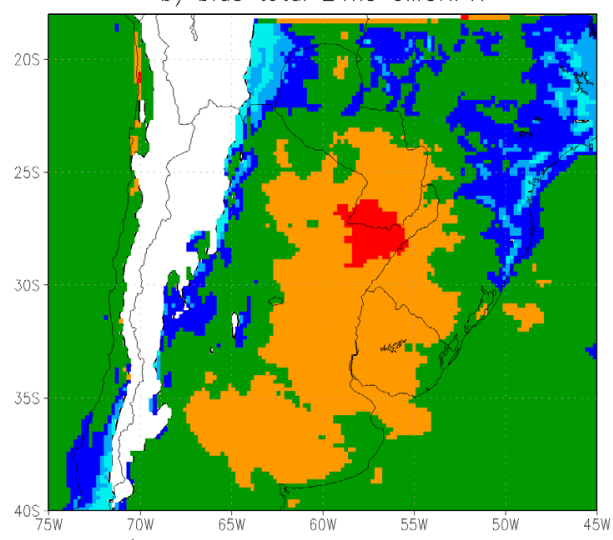
d) bias total $24 \mathrm{hs}$ OBSERVACIONES

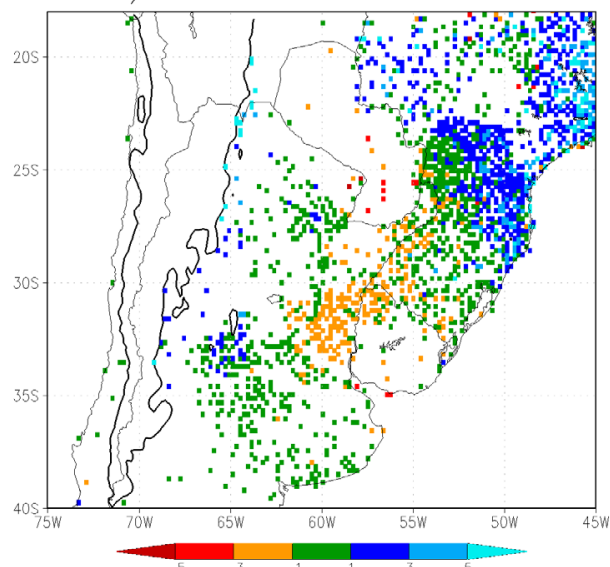

Figura 7 - RMSE y BIAS del pronóstico a 24 hs de precipitación acumulada en 24hs: a) y b) pronósticos respecto de estimaciones CMORPH; c) y d) respecto de observaciones convencionales. Las regiones con topografía mayor a 1500m donde no se realizan los cálculos se indican en blanco con contornos negros. En los campos de observaciones el blanco representa falta de dato. 
Brasil asociada con la Zona de convergencia del Atlántico Sur (SACZ) .Este comportamiento en las simulaciones también es observado por Ruiz et al. (2009), donde se destaca un déficit en la precipitación sobre la región a la salida del SALLJ y un exceso sobre el área de la SACZ. .

En la Figura 8 se muestran los campos de BIAS y RMSE de los pronósticos a 24 horas respecto a los datos CMORPH separados en el semestre frío y cálido. Si bien se observa en ambos semestres que el modelo subestima la precipitación pronosticada en el Noreste de Argentina, dicha subestimación es mucho más importante en el semestre cálido cuando la precipitación es más intensa en la región y existe una relación más notable con la ocurrencia del SALLJ (Nicolini y Saulo, 2006, Salio y Nicolini, 2006, Salio et al., 2007). Como se mostró previamente el modelo no logra representar correctamente el SALLJ en Resistencia lo que puede estar contribuyendo al error que se observa en la precipitación en la región Noreste de Argentina.

Con el fin de evaluar si la subestimación de la precipitación en el noreste de Argentina se debe a unos pocos casos extremos o señala una característica que se repite en numerosos casos, se estudia la evolución temporal de la precipitación diaria pronosticada por el modelo y estimada mediante CMPORH. Se considera el valor medio diario en el área comprendida entre 26 y $31^{\circ} \mathrm{S}$ de latitud y 55 y $60^{\circ} \mathrm{O}$ de longitud, que representa el área donde la subestimación es mayor (El área se indica en la Figura 1). En la Figura 9 se muestra la evolución de la precipitación media diaria estimada y la diferencia entre el pronóstico a 24 horas y la estimación. Se puede observar que en la mayor parte de los días existe una subestimación de la precipitación, concentrándose los valores más importantes en los meses cálidos, y mostrando que no es una característica de algunos pocos casos extremos.

En particular, se analizan todos los casos en los que la precipitación areal media diaria observada, (en el área donde la subestimación es mayor, indicada en la Figura 1), supera los $20 \mathrm{~mm}$ y la subestimación del pronóstico en algún punto del área es mayor a $30 \mathrm{~mm}$, que representan 14 casos sobre un total de aproximadamente 900 días. En estos 14 casos se pronostica la precipitación pero en menor intensidad o desplazada espacialmente. En algunos de estos casos si bien se alcanza el a) rmse semestre calido $24 \mathrm{hs}$

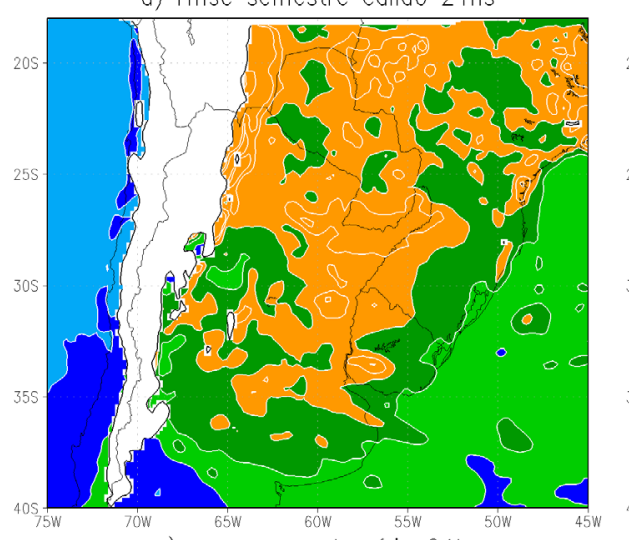

c) rmse semestre frio $24 \mathrm{hs}$

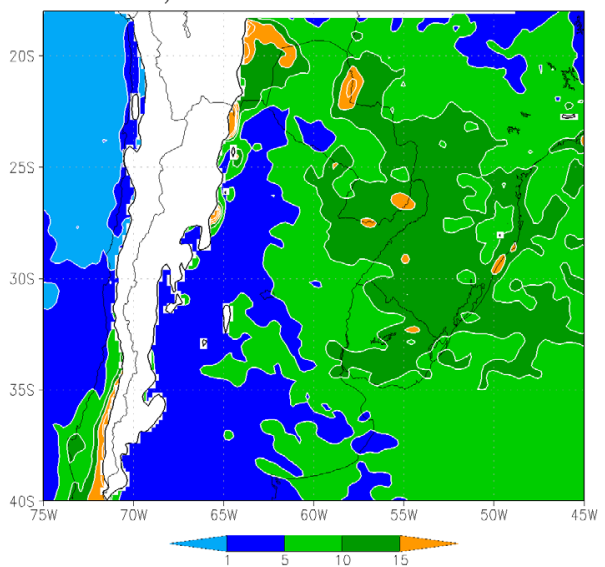

b) bias semestre calido $24 \mathrm{hs}$

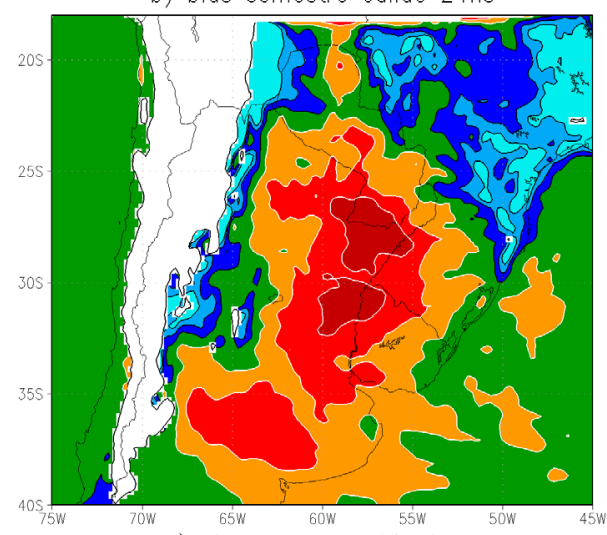

d) bios semestre frio $24 \mathrm{hs}$

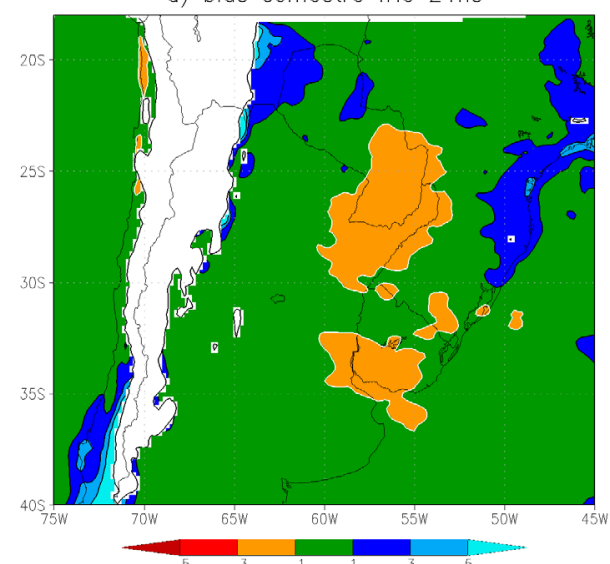

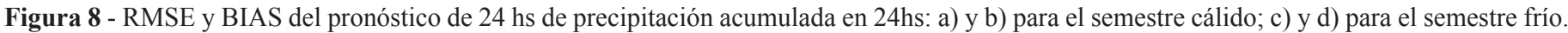
Las regiones con topografía mayor a $1500 \mathrm{~m}$ donde no se realizan los cálculos se indican en blanco con contornos negros. 
valor máximo de precipitación estimado mediante CMORPH, el área que abarca el máximo es menor en el pronóstico, por lo tanto la precipitación areal resulta menor.

En la Figura 10 se presenta la distribución espacial de frecuencia de días sobre el total del periodo en que la precipitación diaria superó un umbral de $1 \mathrm{~mm}$ y uno de $50 \mathrm{~mm}$ respectivamente, con el fin de evaluar en el primer caso si el fenómeno de "lluvia - no lluvia" esta bien pronosticado y en el segundo caso si el modelo logra captar los casos de precipitación extrema. Se puede ver nuevamente que para el umbral de $50 \mathrm{~mm}$, el modelo

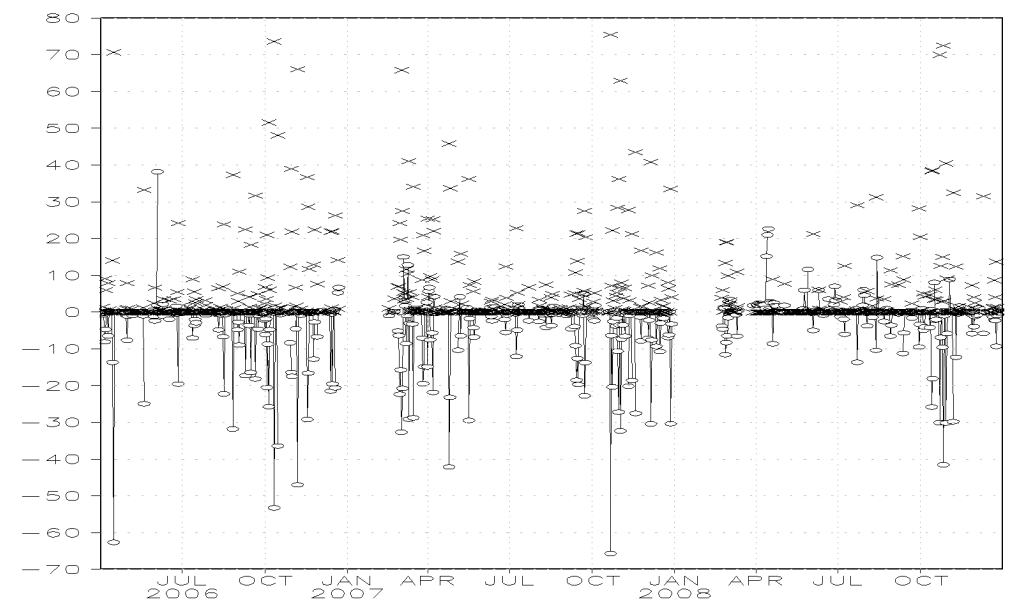

Figura 9 - Precipitación acumulada de 24 horas promediada en el área comprendida entre 26 y $31^{\circ} \mathrm{S}$ de latitud y 55 y $60^{\circ} \mathrm{O}$ de longitud: estimada según CMORPH (cruces) y diferencia entre el pronóstico de 24 horas y la estimación CMORPH (círculos con líneas).
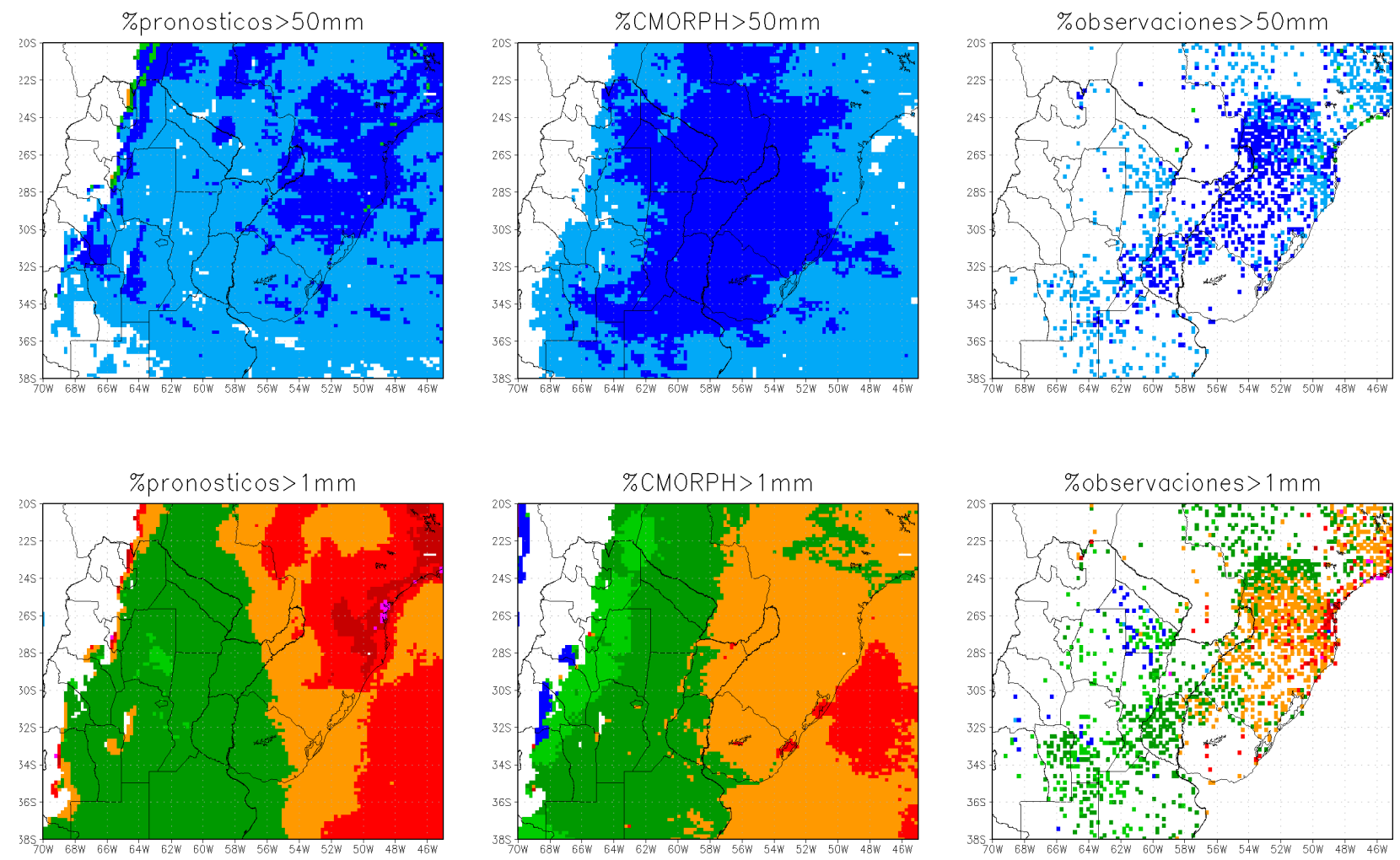

Figura 10 - Porcentaje de días sobre el total en los que la precipitación acumulada de 24hs superó los 50 mm y 1 mm, paneles superiores e inferiores respectivamente. De izquierda a derecha pronósticos, CMORPH y observaciones convencionales. 
muestra subestimaciones especialmente en la región noreste de Argentina, la cantidad de casos con precipitación extrema, tanto al compararlos con las estimaciones CMORPH como frente a las observaciones. La débil representación del SALLJ debilita la convergencia del flujo de vapor de agua y por ende debilita la formación de sistemas convectivos intensos que son los generadores del $80 \%$ de las precipitaciones en la región (Salio et al., 2007; Zipser et al., 2006). En cambio, para el umbral de $1 \mathrm{~mm}$, si bien en Argentina la detección de casos de lluvia es buena, se observa una gran sobreestimación de casos de lluvia en el sudeste de Brasil, posiblemente relacionados con la topografía de la región, dado la dificulta que presentan los modelo en general para pronosticar la precipitación en zonas con topografía compleja.

La pregunta que surge es cuáles son las causas que pueden estar generando la subestimación de la precipitación en la región noreste de Argentina. Si bien se observó en los perfiles de BIAS de la temperatura una estabilización del gradiente. Este hecho se produce por igual en todas las localidades y la subestimación en la precipitación no es de la misma magnitud en toda la región. Se encontró también una subestimación de la humedad y de la componente meridional del viento en Resistencia lo que esta indicando una falencia en la representación del SALLJ que es uno de los mecanismos responsables de la precipitación en la región.

Con el fin de analizar en mayor detalle la capacidad del modelo para pronosticar el SALLJ, se realiza una composición de los campos asociados al mismo, correspondiente a todos los días que verifican los requerimientos de la definición de casos SALLJ en los análisis GFS, de acuerdo a Salio et al. (2002) y Nicolini et al. (2005c). Estos trabajos consideran una intensidad del viento superior a $12 \mathrm{~m} / \mathrm{s}$, una cortante del viento entre 850 y $700 \mathrm{hPa}$ superior a $6 \mathrm{~m} / \mathrm{s}$ y que el viento deber ser predominantemente del norte en el área cercana a Santa Cruz de la Sierra Bolivia $\left(17^{\circ} \mathrm{S}-63^{\circ} \mathrm{W}\right)$. Se muestra en la Figura 11, la composición del viento en $850 \mathrm{hPa}$, el flujo de humedad integrado en la vertical y la convergencia del mismo, calculada con los pronósticos a 24 horas del BRAMS y con los análisis de GFS, ambos válidos para las 12 UTC. Se puede apreciar en la figura que la intensidad del SALLJ esta subestimada en los pronósticos y la zona de convergencia del vapor de agua
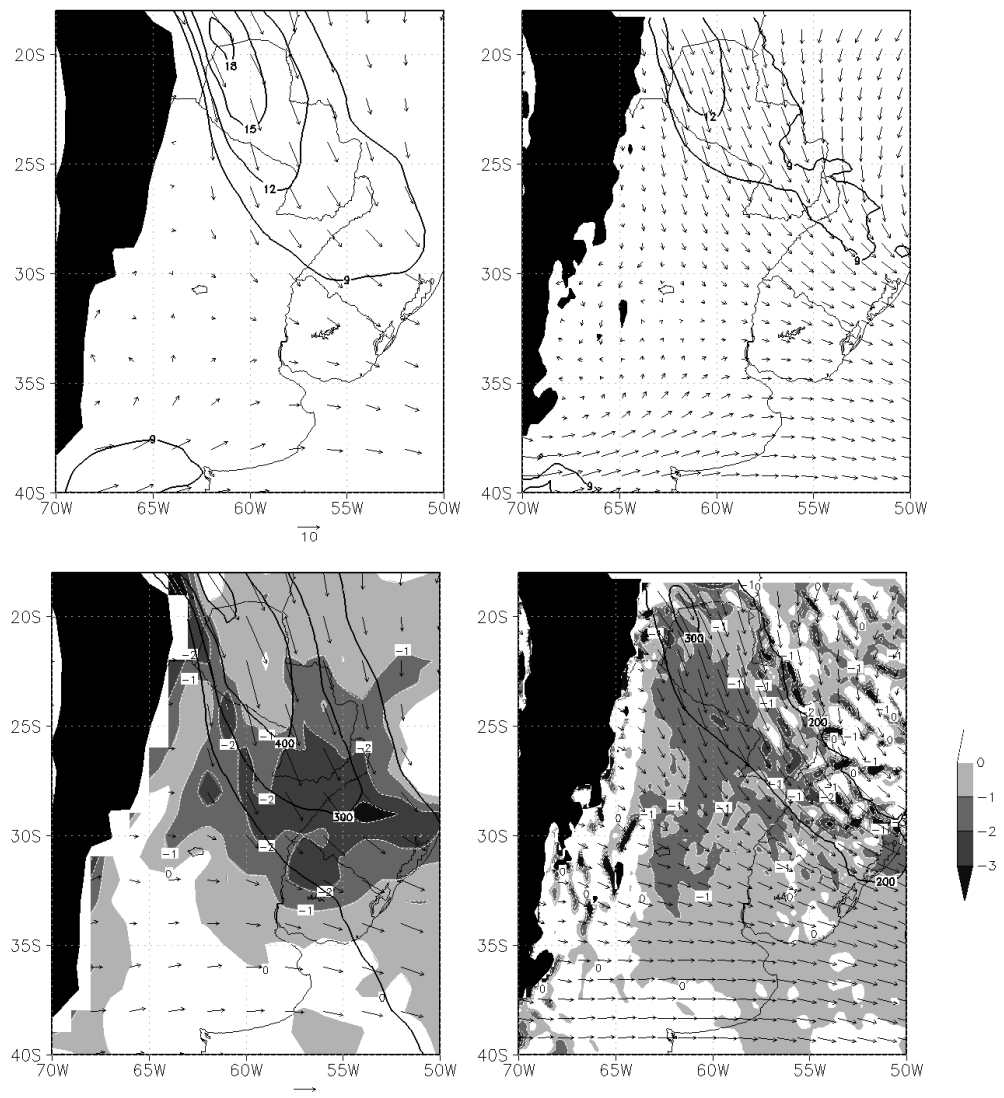

Figura 11 - Viento (vectores) e intensidad (contornos) en $850 \mathrm{hPa}$ en $\mathrm{m} / \mathrm{s}$ (paneles superiores). Flujo de vapor de agua integrado en la vertical entre 1000 y $300 \mathrm{hPa}$ ( vectores en kg m-1 s-1), su intensidad (contornos) y su divergencia (sombreado) (paneles inferiores), para el análisis del GFS (paneles izquierdos) y el pronóstico a $24 \mathrm{hs} \mathrm{del} \mathrm{BRAMS} \mathrm{(paneles} \mathrm{derechos)} \mathrm{válido} \mathrm{para} \mathrm{las} 12$ UTC para eventos SALLJ. Las áreas de montaña han sido enmascaradas para topografías superiores a $1500 \mathrm{~m}$. 
se encuentra desplazada al oeste y subestimada en magnitud. Estas composiciones nuevamente señalan una dificultad del modelo para representar tanto la intensidad como la ubicación del máximo del SALLJ. Dado dicha falencia en la representación del SALLJ es de esperar una subestimación de la precipitación asociada al mismo.

Las fallas en la representación del SALLJ pueden tener múltiples causas tanto asociadas a la representación del ciclo diurno de la capa límite planetaria, la topografía y/o la representación de la liberación del calor latente. Por otro lado, Saulo et al. (2007) encuentran mediante experimentos de sensibilidad utilizando el modelo RAMS que el SALLJ es reforzado por la liberación de calor latente en la convección organizada en su parte delantera. Una correcta predicción del mecanismo de retroalimentación entre el SALLJ y la convección generadora de precipitación en la región, propuesto por estos autores, requiere un pronóstico correcto del transporte de humedad, vehiculizado en niveles bajos de la troposfera fundamentalmente por el SALLJ. Una subestimación de la intensidad del SALLJ y de la convergencia de flujo de humedad asociada conducen a un debilitamiento de este mecanismo.

Una alternativa a estudiar para mejorar el pronóstico de precipitación en dicha región es la posibilidad de extender el dominio de mayor resolución hacia el norte de forma de poder representar el SALLJ con mayor exactitud, ya que la región de Bolivia donde se produce su máximo no está incluida en el dominio de mayor resolución. A la vez, al producirse una sobreestimación de la precipitación en la región asociada a la SACZ, esto puede estar contribuyendo a la subestimación de la precipitación al sur de la misma (Liebmann et al., 2004).

\section{CONCLUSIONES}

En el presente trabajo se logra documentar mediante diferentes estadísticos el desempeño del modelo BRAMS en el sur de Sudamérica para un período de aproximadamente 3 años. La verificación realizada muestra un desempeño del modelo BRAMS similar al de otros modelos de la misma resolución utilizados en la región (Saulo et al., 2008; Ruiz et al.,2010). Asimismo en el presente trabajo se señalan algunas deficiencias del modelo que es necesario indagar en mayor detalle de forma de poder ajustar su configuración con el fin de mejorar su desempeño en la región.

Se evaluaron los perfiles verticales de temperatura, humedad y componentes del viento pronosticado frente a las observaciones disponibles. Se destaca una tendencia a pronosticar perfiles de temperatura más estables y con un mayor contenido de humedad que los observados. A la vez se detecta cierta falencia en la representación SALLJ. Este hecho se observa en los perfiles verticales de viento y humedad en la localidad de Resistencia, y también en las composiciones de los casos SALLJ frente a los análisis GFS, donde se muestra una subestimación del máximo del SALLJ y de la convergencia de humedad asociada al mismo. En cuanto a la precipitación se señala la dificultad que presenta el modelo para representar la precipitación en el noreste de Argentina, especialmente en el semestre cálido, mostrando una importante subestimación de la misma, lo que puede estar relacionado con la falta de precisión en el pronóstico del SALLJ. Resulta de interés para trabajos futuros el estudio en mayor detalle de cuales son los mecanismos que el modelo está representando erróneamente y que conducen a dicha subestimación, realizando experimentos de sensibilidad con el modelo.

Si bien existen distintas verificaciones de los modelos regionales que actualmente se aplican en la región con una resolución similar, como el WRF del CIMA y el ETA del Servicio Meteorológico Nacional, resulta difícil realizar una comparación estricta entre los estadísticos debido principalmente a que no coinciden los conjuntos de datos utilizados, el período considerado y la metodología aplicada. Se plantea el interés de realizar en trabajos futuros una comparación del desempeño de los distintos modelos.

Además es importante señalar que nuevamente se pone de manifiesto la necesidad de contar con un mayor número de observaciones en la región a la hora de realizar una verificación, especialmente para variables como la precipitación. Si bien la estimación de precipitación CMORPH en cierta forma cubre dicha necesidad, este conjunto de datos presenta algunas deficiencias y requiere aún de un mayor avance en la técnica de la generación de la estimación de la precipitación observada.

\section{AGRADECIMIENTOS}

Los autores agradecen a las siguientes instituciones por proveer los datos de precipitación observada para realizar el presente trabajo: Servicio Meteorológico Nacional, Administración Provincial del Agua (Chaco), Secretaría de Recursos Hídricos de la Nación, Universidad de La Punta (San Luis), Autoridad Interjurisdicional del Aguas (Neuquén), Comisión Técnica Mixta Salto Grande, Instituto Nacional de Tecnología Agropecuaria y Bolsa de Cereales de Argentina; Dirección Nacional de Meteorología de Uruguay y de Paraguay, Centro de Previsão de Tempo e Estudos Climáticos de Brasil; y Climate Diagnostics Center (CDC)-NOAA de E.E.U.U.. Esta investigación fue financiada mediante los proyectos de ANPCYT: PICT2007-00355, PICT2008-215 y 1603, UBACyT X159, CONICET PIP -5582, PIDDEF 2010/47. El trabajo se pudo realizar gracias a la beca post-doctoral de CONICET otorgada a la primera autora. 


\section{REFERENCIAS}

BERBERY, H.; V. BARROS. The hydrologic cycle of the La Plata Basin in South America. Journal of Hydrometeorology, v. 3, p. 630-645, 2002.

BORQUE, P.; P. SALIO, M. NICOLINI; Y. GARCÍA SKABAR. Environment Associated with Deep Moist Convection under SALLJ Conditions: A Case Study. Weather and Forecasting, v. 25, p. 970-984. doi: 10.1175/2010WAF2222352.1, 2010.

CHEN, C.; W. R. COTTON. A one dimensional simulation of the stratocumulus-capped mixed layer. Boundary-Layer Meteorology, v. 25, p. 289-321, 1983.

DAVIES, J.C. Statistics and Data Analysis in Geology. J. Wiley Ed., New York, 550pp, 1973.

EBERT, E.; J.E. JANOWIAK; C. KIDD. Comparison of Near-Real-Time Precipitation Estrimates from Satellite Observations and Numerical Models. Bulletin of the American Meteorological Society, v. 88, p. 47-64, 2007.

FREITAS, S. R. et al. The Coupled Aerosol andTracer Transport model to the Brazilian developments on the Regional Atmospheric Modeling System (CATT-BRAMS). Part 1: Model description and evaluation. Atmospheric Chemistry and Physics, v. 9, p. 2843-2861, 2009.

GARCÍA SKABAR, Y. Análisis enriquecidos, evaluación de su impacto en el pronóstico y su aplicación para describir circulaciones de mesoescala en el centro y norte de Argentina. Tesis doctoral, DCAO, FCEyN, UBA. pp. 177, 2008.

GARCÍA SKABAR, Y; M. NICOLINI. Enriched Analyses with Assimilation of SALLJEX Data. Journal of Applied Meteorology and Climatology, v. 48, p. 2425-2440. doi: 10.1175/2009JAMC2091.1, 2009.

GEVAERD, R.; S.R. FREITAS. Estimativa operacional da umidade do solo para iniciação de modelos de previsão numérica da atmosfera parte I: descrição da metodologia e validação. Revista Brasileira de Meteorología, v. 31, n. 3, p. 59-73, 2006.

GRELL, G. A. Prognostic evaluation of assumptions used by cumulus parametrizations. Monthly Weather Review, v. 121, p. 764-787, 1993.

GRELL, G.A.; D. DEVENYI. A generalized approach to parameterizing convection combining ensemble and data assimilation techniques. Geophysical Research Letters, v.29, n. 14, p. 1963, 2002.

HUFFMAN G. J.; R. F. ADLER; D. T. BOLVIN; G. GU, E. J. NELKIN; Y. HONG; D. B. WOLFF; K. P. BOWMAN; E. F. STOCKER. The TRMM multisatellite analysis (TMPA): Quasi-global multiyear, combined-sensor precipitation estimates at fine scales. Journal of Hydrometeorology, v. 8, p. 38-55, 2007.
JOYCE, R. J.; J. E. JANOWIAK; P. A. ARKIN; P. XIE. CMORPH: A method that produces global precipitation estimates from passive microwave and infrared data at high spatial and temporal resolution. Journal of Hydrometeorology, v. 5, p. 487-503, 2004.

LIEBMANN, B.; D. ALLURED. Daily Precipitation Grids for South America. Bulletin of the American Meteorological Society. v. 86, n. 11, p. 1567-1570, 2005.

LIEBMANN, B., G. N. KILADIS, C. S. VERA, A. C. SAULO, L. M. V. CARVALHO. Subseasonal Variations of Rainfall in South America in the Vicinity of the Low-Level Jet East of the Andes and Comparison to Those in the South Atlantic Convergence Zone. Journal of Climate, v. 17, p. 38293842.doi: 10.1175/1520-0442(2004)017, 2004.

MARENGO, J; W. SOARES; C. SAULO; M. NICOLINI. Climatology of the LLJ east of the Andes as derived from the NCEP reanalyses. Journal of Climate, v. 17, p. 22612280, 2004.

MELLOR G.L.; T. YAMADA. Development of a turbulence closure model for geophysical fluid problems. Reviews of Geophysics and Space Physics, v. 20, p. 851-875, 1982.

MESINGER, F. Improvements in quantitative precipitation forecast with the ETA regional model at the National Centers for Environmental Prediction: The $48 \mathrm{~km}$ upgrade. Bulletin of the American Meteorological Society, v. 77, p. 2637-49, 1996.

NICOLINI, M.; A. C. SAULO. Modeled Chaco low-level jets and related precipitation patterns during the 1997-1998 warm season. Meteorology and Atmospheric Physics, v. 94, p. 129-143, 2006.

NICOLINI, M.; Y. GARCÍA SKABAR; A.G. ULKE; A. C. SAULO. RAMS model performance in simulating precipitation during strong poleward low level jet events over northeastern Argentina. Meteorologica - Special Issue on Variability of the South American Monsoon System, v 27, n. 1 y 2, p. 89-98, 2002.

NICOLINI, M.; Y. GARCÍA SKABAR, A.G. ULKE; P. SALIO. Simulación de una tormenta granicera en Mendoza. Actas del IX Congreso Argentino de Meteorología organizado por el Centro Argentino de Meteorólogos. Buenos Aires, Argentina. 2005a.

NICOLINI, M.; M. TORRES BRIZUELA; Y. GARCÍA SKABAR: Simulación de una tormenta tornádica usando un modelo de mesoescala de alta resolución. Actas del IX Congreso Argentino de Meteorología organizado por el Centro Argentino de Meteorólogos. Buenos Aires, Argentina. 2005b.

NICOLINI M., P. SALIO Y P. BORQUE: Caracterización de la estructura vertical térmica y cinemática de la troposfera baja en el Norte Argentino durante el SALLJEX. Actas 
del IX Congreso Argentino de Meteorología organizado por el Centro Argentino de Meteorólogos. Buenos Aires, Argentina. 2005c.

NICOLINI, M.; Y. GARCÍA SKABAR. Diurnal cycle in convergence patterns in the boundary layer east of the Andes and convection, Atmospheric Research, v. 100, n. 4, p. 377-390, 2011.

REYNOLDS, R.W.; N.A. RAYNER; T.M. SMITH; D.C. STOKES; W. WANG. An Improved In Situ and Satellite SST Analysis for Climate, Journal of Climate, v. 15, 2002.

ROBLEDO, F.; O. PENALBA. Temporal and spatial variability of daily intensity rainfall and it relation with surface atmospheric circulation in Southeastern South America. 9th International Conference on Southern Hemisphere Meteorology and Oceanography.(9ICSHMO). 9-13 February 2009, Melbourne, Australia. 2009.

RUIZ J.J. Evaluation of different methodologies for precipitation estimates calibration CMORPH - over South America. Revista Brasileira de Meteorología, v. 24, n. 4, p. 473488, 2009.

RUIZ,J.J.; A. C. SAULO; E. KALNAY. Comparison of Methods Used to Generate Probabilistic Quantitative Precipitation Forecasts over South America. Weather and Forecasting, v. 24, p. 319-336, 2009.

RUIZ,J.J.; A. C. SAULO; J. NOGUES-PAEGLE WRF Model Sensitivity to choice of Parameterization over South America: Validation against Surface Variables. Monthly Weather Review, v. 138, p. 3342-3355, 2010.

RUIZ, J.J.; A. C. SAULO; Y. GARCÍA SKABAR; P. V. SALIO. Evaluación de la representación de un sistema convectivo de mesoescala utilizando el modelo RAMS. Meteorologica, v. 31, n. 1y 2, 13-35, 2006

SALIO, P., C. CAMPETELLA, J. RUIZ, Y. GARCÍA SKABAR Y M. NICOLINI. Nevada en el sudeste Bonaerense: Climatología sinóptica y un caso de estudio. Meteorologica, v. 31, n. 1y 2, 67-84, 2006.

SALIO, P.; D. VILA; Y. GARCIA SKABAR; M. P.HOUBOUCHIAN;C. MATSUDO, 2010: Performance evaluation of Precipitation Estimations over South-Eastern South America considering different climatic regions. 5th IPWG Workshop on Precipitation Measurements, Hamburg, 11-15 Octubre, 2010.

SALIO, P.; M. NICOLINI. Seasonal characterization on the diurnal cycle of convection frequency over Southeastern South America under different low-jet conditions. 8th International Conference on Southern Hemisphere Meteorology and Oceanography (8ICSHMO). Foz do Iguaçu, Brazil, April, 24-28, 2006, p. 1157-1162, 2006.

SALIO, P.; M. NICOLINI; A.C. SAULO. Chaco Low-Level Jet Events Characterization During the Austral Summer Season by ERA Reanalysis. Journal of Geophysical Research Atmosphere, v. 107, D. 24. 10.1019/2001JD001315, 2002. SALIO, P.; M. NICOLINI; E. J. ZIPSER. Mesoscale Convective Systems over Southeastern South America and their relationship with the South American Low Level Jet. Monthly Weather Review, v. 135, n. 4, p. 1290-1309, 2007. SALIO, P.; Y. GARCÍA SKABAR; M. NICOLINI. "The role of the low level jet in a flash flood event over central Argentina". Actas de la "5th European Conference on Severe Storms, ECSS 2009”, Landshut, Alemania. On line: http:// www.essl.org/ECSS/2009/preprints/P03-03-salio.pdf. 2009.

SAULO, C.; J.J. RUIZ; Y. GARCÍA SKABAR. Synergism between the low level jet and organized convection at its exit region. Monthly Weather Review, v. 135, p. 1310-1326, ISSN: 0027-0644, 2007.

SAULO, A.C.; L. FERREIRA. Evaluation of quantitative precipitation forecast over southern South America. Australian Meteorological Magazine, v. 52, p. 81-93, 2003.

SAUlO,A.C., M. SEluCHI, C.CAMPETELLA, L. FERREIRA. Error evaluation of NCEP and LAHM regional model daily forecasts over Southern South America. Weather and Forecasting, v. 16, n. 6, p. 697-712, 2001.

SAULO, C.; S. CARDAZZO; J. RUIZ; C. CAMPETELLA; A. ROLLA. El sistema de pronóstico experimental del Centro de Investigaciones del Mar y la Atmósfera, Meteorologica, v. 33, p. 83-97, 2008.

SMAGORINSKY, J., 1963: General circulation experiments with the primitive equations. Part I, The basic experiment. Monthly Weather Review, v. 91, p. 99-164, 1963.

SOUZA, E. P. ; E. M. SILVA. Impacto da Implementação de uma Parametrização de Convecção Rasa em um Modelo de Mesoscala. Descriçao e Testeo de Sensibilidade do Esquema. Revista Brasileira de Meteorologia, v. 18, n. 1, p. 33-42, 2002.

TRIPOLI, G. J.;W. R. COTTON. The Colorado State University three dimensional cloud/mesoscale model- 1982. Part I: General theoetical framework and sensitivity experiments. Journal de Recherches Atmospheriques, v. 16, p. 185220, 1982.

WALKO, R. L.; W. R. COTTON; M. P. MEYERS; J. Y. HARRINGTON. New RAMS cloud microphysics parameterization: Part I. The single-moment scheme. Atmospheric Research, v.38, 29\u201362, 1995.

ZIPSER, E.J.;C. LIU; D.J. CECIL; S.W. NESBITT; D. P. YORTY. Where are the most intense thunderstorms on earth?. Bulletin of the American Meteorological Society, v. 87, n. 8 , p. $1057-1071,2006$. 